\author{
Cesar A. Garcia-Franco \\ David W. Mead
}

\section{Rheological and molecular characterization of linear backbone flexible polymers with the Cole-Cole model relaxation spectrum}

Received: 3 March 1998

Accepted: 30 September 1998

\begin{abstract}
The empirical Cole-Cole distribution is an analytical three parameter model of the relaxation spectra that provides accurate fits to experimental dynamic viscosity data for many systems of commercial linear backbone flexible polymers. We demonstrate that for disparate systems of polyethylenes, the three Cole-Cole model parameters have simple power law relationships to moments of the molecular weight distribution enabling direct molecular interpretation of the mechanical relaxation spectrum. A simple relationship between the Cole-Cole distribution and the Cross model for the non-linear flow curve can be deduced utilizing the empirical CoxMerz rule. Accurately representing
\end{abstract}

the linear viscoelastic material functions with empirical analytical relaxation spectra containing relatively few fitting parameters that can be readily interpreted is a major advance in polymer characterization. The three Cole-Cole parameters effectively replace single point material characterizations such as melt flow index. Development of higher resolution polymer characterization methods is imperative with the advent of metallocene catalyst technology, which enables the molecular weight and backbone architecture to be carefully controlled.

Key words Polyethylene - ColeCole distribution - Metallocene Ziegler - Viscosity

\section{Introduction}

Quantitative characterization of the processing and molecular properties of commercial polymers has long been an area of active research interest, both industrially (Zeichner and Patel 1982) and academically (Burke and Weiss 1975). The principal objective of any characterization method is to capture the salient features of a complex system in a finite number of parameters that can be readily interpreted and extrapolated to general situations. Industrially, empirical methods such as the melt flow index (MFI) (ASTM D1238) are still widely used to characterize the rheological properties of resins. The MFI is a simple single point method that completely neglects the complex time dependence of polymers. Although empirical correlations of the MFI with molecular parameters exist, the correspondence is often obscured by the non-homogeneity of the flowfield and the possibility that the stress level is sufficiently high that non-linearities in the flow curve are important (Bremner et al. 1990; Shenoy et al. 1984).

With the advent of metallocene catalyst technology, it is now possible to produce polymers with carefully controlled molecular weight, molecular weight distribution (MWD) and backbone architecture in commercially relevant scales (Huang and Rempel 1995; Thayer 1995) These vastly improved chemical capabilities have outstripped the resolution of conventional molecular weight characterization methods such as Gel Permeation Chromatography GPC and intrinsic viscosity (Burke and Weiss 1975; Nguyen et al. 1996). In order to fully exploit the advances in polymer catalyst technology, additional rheological, molecular weight and backbone architecture characterization methods must be 
developed. Methods such as MFI, GPC and intrinsic viscosity simply do not have the resolution to distinguish subtle differences in the molecular and rheological properties of metallocene catalyzed polymers, particularly for the high molecular weight tail of the distribution. However, since most characterization methods must be used on a routine basis in industrial settings, simplicity with respect to both experimentation and interpretation is absolutely required.

This work is directed at developing enhanced resolution material characterization methods for metallocene catalyzed polymers. Rheological methods are particularly well suited for this task by virtue of their inherent sensitivity to molecular weight, MWD and backbone architecture (Graessley 1982; Mead 1996). To satisfy the constraint of simplicity with respect to interpretation and experimentation, we shall focus on analytical models of linear viscoelastic behavior in the terminal and plateau regions of the relaxation spectrum.

The status of quantitative molecular structure-rheological property relationships was advanced dramatically by the seminal work of des Cloizeaux (1988) and Tsenoglou $(1987,1991)$ on the "double reptation" mixing rule. For linear backbone flexible polymers, the double reptation mixing rule provides an accurate, quantitative relationship between the linear viscoelastic material properties and the MWD. The mathematical relationship is sufficiently simple that when the linear viscoelastic material functions are known, it can be inverted to yield the underlying MWD (Mead 1994; Wasserman 1995). This inversion procedure is a relatively new application of linear viscoelastic measurements, analytical rheology, i.e. using melt rheology as a method of analytical chemistry. There is considerable precedent for this application in the sense that intrinsic viscosity is an analytical technique based on dilute solution rheological behavior of polymers.

A detailed explanation of why the relatively simple theoretical description contained in the double reptation model works as well as it does for a problem as complex as polydispersity has recently been published by Milner (1996). A more expansive and thorough description of the molecular dynamics of polydisperse systems can be found in Viovy et al. (1991). Although the double reptation model is currently the most widely used mixing rule for linear backbone flexible polymers, we shall seek mathematically simpler models that contain essentially equivalent information. In this regard, we shall be examining the Cole-Cole model relaxation spectrum which for a wide array of linear flexible polymers provides an accurate description of the plateau and terminal linear viscoelastic properties. In a subsequent paper, we shall demonstrate that for linear backbone flexible polymers with a log-normal or Schultz distribution, the Cole-Cole relaxation spectrum with appropriately chosen parameters is functionally equivalent to the double reptation model. Thus, our primary motivation for developing the Cole-Cole method is the ease with which material characterization can be performed relative to working directly with the double reptation model (Mead 1994; Wasserman 1995). We are particularly interested in interpreting the Cole-Cole model parameters in terms of fundamental characteristics of the MWD.

This paper demonstrates the viability of the ColeCole model as a rheological and molecular characterization tool that is experimentally simple while greatly enhancing the sensitivity and resolution with respect to the molecular and processing characteristics of the resin. The enhanced resolution is a direct consequence of fully incorporating the complex time dependence of the material response into the characterization. This paper will mollify the view that the generally observed agreement of the Cole-Cole model relaxation spectrum with experimental data for many commercial resins is a suspicious coincidence rather than the powerful yet mathematically simple tool to characterize the flow curve and MWD of commercial polymer that it truly is.

This paper is organized in the following manner; in the second section we review the origins of the empirical Cole-Cole model for both the dielectric relaxation spectrum and the mechanical relaxation spectrum. In the third section the Cole-Cole model is applied to dynamic moduli data of three families of polyethylene resins. The model parameters are calculated and correlated with moments of the MWD. In the fourth section we explore the relationship between the Cole-Cole model and the Cross model for the flow curve thus establishing a direct link with non-linear flow properties.

\section{The Cole-Cole model relaxation spectrum}

The empirical Cole-Cole model spectrum was first introduced in the context of the dielectric relaxation spectrum, $\varepsilon^{*}(\omega)$, of ordinary low molecular weight liquids (Cole and Cole 1941) and continues to be used in this context (Carrique and Delgado 1996). The model is empirical and has the following structure;

$\varepsilon^{*}(i \omega)=\frac{\varepsilon_{0}-\varepsilon_{\infty}}{1+(i \omega \lambda)^{(1-\alpha)}}+\varepsilon_{\infty}$

Here, $\omega$ is the angular frequency, $\lambda$ is a characteristic relaxation time, $\alpha$ is the dispersion parameter, $\varepsilon_{0}$ and $\varepsilon_{\infty}$ are the asymptotic dielectric constants. Although the Cole-Cole spectrum was first introduced in the context of the dielectric relaxation spectrum of low molecular weight liquids, it was subsequently applied to the dielectric spectrum of polymeric materials by Havriliak and Negami (1967). These authors realized that the molecular mechanism underlying dielectric relaxation and stress relaxation in polymers were identical. Specifically, the same molecular motions that generate a frequency dependence for the dielectric spectrum are 
also responsible for the relaxation of orientation in polymeric liquids (McCall 1969; Taylor et al. 1985). In fact, this fundamental concept has been used to extract MWD information from the dielectric spectrum (Fodor and Hill 1994). With these ideas in mind, Havriliak and Negami (1967) extended the Cole-Cole type spectrum to the linear viscoelastic mechanical properties of polymer melts, specifically the dynamic compliance and dynamic viscosity functions. The Cole-Cole expression for the complex dynamic viscosity, $\eta^{*}$, has the following form (Labaig et al. 1973; Lanfray 1988),

$\eta^{*}(i \omega)=\frac{\eta_{0}}{1+\left(i \omega \lambda_{0}\right)^{(1-\alpha)}}$

As before, $\omega$ is the angular frequency, $\lambda_{0}$ is a characteristic relaxation time, $\alpha$ is the dispersion parameter, and $\eta_{0}$ is the zero shear viscosity. For many systems of mono-modal linear backbone flexible polymer, the ColeCole model provides an accurate fit to experimental data over most of the frequency range with only three parameters. Note that the Cole-Cole model does not correctly predict asymptotic terminal behavior; specifically the slopes of the storage and loss modulus do not approach 2 and 1 on a log-log plot. However, for most commercial polymers asymptotic terminal behavior is typically difficult to realize experimentally. There is no detailed molecular model associated with the Cole-Cole model. The principal basis for the model is that its mathematical structure is such that only three undetermined parameters are required to capture the salient features of the relaxation spectrum. The numerical procedure for determining the three model parameters from dynamic moduli data is outlined in the appendix.

\section{Application of the Cole-Cole model relaxation spectra to commercial polymers}

In this section we examine the linear viscoelastic relaxation spectra of several commercial polyethylene resins in the framework of the Cole-Cole analysis presented above. Specifically, we shall examine the quality of the fit of the Cole-Cole distribution to experimental dynamic viscosity data. Subsequently, we correlate the model parameters with moments of the MWD.

The specific linear flexible polymer systems we shall investigate in this study can be separated into three families based on their catalyst parentage. The catalyst system and the polymerization conditions determine the molecular structure, principally the shape of the MWD and mean molecular weight. Differences in catalyst chemistry and/or polymerization conditions are manifested directly in the shape of the MWD. In this regard, detailed knowledge of the catalyst system and the underlying kinetics has long been used to construct model MWDs such as the Flory "most probable" distribution for ideal condensation polymerizations.

The first catalyst family we shall study consists of anionically polymerized polyethylenes with very narrow MWDs and a variety of molecular weights. The second family is comprised of gas phase metallocene catalyzed polyethylenes spanning a range of molecular weights. The MWDs for such systems are relatively narrow $\left(M_{\mathrm{w}} / M_{\mathrm{n}} \sim 2\right)$. The final polymer system consists of gas phase Zeigler-Natta catalyzed polyethylenes with a relatively broad MWDs $\left(M_{\mathrm{w}} / M_{\mathrm{n}} \sim 4^{+}\right)$. For a given catalyst system, the shape of the MWD will be very similar from polymer to polymer, the principal distinction coming from a horizontal shift of the MWD along the molecular weight axis. The molecular characteristics of the materials in our study are summarized in Table 1 . Within a given catalyst family, the Cole-Cole dispersion parameter $\alpha$ is found to be constant.

In Fig. 1 we examine the complex viscosity of a metallocene polymer (M5) on a Cole-Cole plot, i.e. a plot of the imaginary dynamic viscosity versus the real dynamic viscosity. Cole-Cole plots have the advantage that the explicit frequency dependence has been eliminated. For linear backbone flexible polymers with a monomodal MWD, Cole-Cole plots yield an arc of a circle. Generally, the broader the MWD the shallower the arc. For a monodisperse system with approximately a single relaxation time, a semi-circle is generated.

Figure 1 is representative of the quality of fit achievable using the Cole-Cole model on commercial polymer resins. Even with linear-linear plots, the fit is still very good. Of particular interest is the quality of the fit in the terminal and plateau region, i.e. at the extreme right of the plot. Also shown in Fig. 1 are calculations of the complex viscosity performed using the double reptation mixing rule with a Schultz MWD with identical $M_{\mathrm{w}} / M_{\mathrm{n}}$ to that measured for the polymer using GPC. The correspondence of the predicted linear viscoelastic material properties with the data is very good, consistent with previously published work (Mead 1994; Wasserman 1995). The discrepancy in the double reptation model predictions and the actual data may be a consequence of the assumed Schultz distribution for the MWD. The principal point here is that the Cole-Cole and double reptation models provide approximately equivalent representations of the experimental linear viscoelasticty data for monomodal systems examined in this paper. Thus, although the Cole-Cole representation is completely empirical, it is able to effectively capture the same information as the quasimolecular double reptation model in a vastly simpler mathematical structure.

Figure 2 plots the relaxation spectra calculated with the experimentally determined Cole-Cole parameters of all catalyst families with the frequency axis normalized with the parameter $\lambda_{0}$. The frequency scaling procedure collapses all the spectra from a given catalyst family 
Table 1 Molecular weight characteristics, Cole-Cole and Cross model parameters of the polyethylene resins studied in this work. The polymers are grouped according to their catalyst parentage; anionic, metallocene and Zeigler-Natta

\begin{tabular}{|c|c|c|c|c|c|c|c|c|c|}
\hline Polymer & $\begin{array}{l}M_{\mathrm{w}} \\
\text { (daltons) }\end{array}$ & $\begin{array}{l}M_{\mathrm{w}} / M_{\mathrm{n}} \\
(-)\end{array}$ & $\begin{array}{l}\eta_{0} \\
\text { (pa-s) }\end{array}$ & $\begin{array}{l}\lambda_{0} \\
\text { (s) }\end{array}$ & $\begin{array}{l}\alpha \\
(-)\end{array}$ & $\begin{array}{l}\eta_{0 c} \\
\text { (pa-s) }\end{array}$ & $\begin{array}{l}\tau \\
(\mathrm{s})\end{array}$ & $\begin{array}{l}m \\
(-)\end{array}$ & $\begin{array}{l}1-m \\
(-)\end{array}$ \\
\hline $\mathrm{A}^{\mathrm{a}}$ & 189000 & 1.13 & 29500 & 0.02 & 0.13 & 28143 & 0.0124 & 1.29 & -0.29 \\
\hline $\mathrm{H}^{\mathrm{a}}$ & 151000 & 1.08 & 10200 & 0.006 & 0.10 & 10148 & 0.0053 & 1.43 & -0.43 \\
\hline $2 \mathrm{LC}^{\mathrm{a}}$ & 245000 & 1.08 & 48000 & 0.05 & 0.12 & 48246 & 0.0291 & 1.26 & -0.26 \\
\hline $\mathrm{B}^{\mathrm{a}}$ & 134000 & 1.07 & 6600 & 0.004 & 0.11 & 6610 & 0.00262 & 1.14 & -0.14 \\
\hline $\mathrm{I}^{\mathrm{a}}$ & 101000 & 1.04 & 2300 & 0.0011 & 0.11 & 2252 & 0.0000201 & 0.48 & 0.52 \\
\hline $2 \mathrm{LD}^{\mathrm{a}}$ & 253000 & 1.04 & 87000 & 0.077 & 0.12 & 87799 & 0.0502 & 1.15 & -0.15 \\
\hline$(\mathrm{M} 1)^{\mathrm{b}}$ & 98000 & 2.61 & 9200 & 0.0398 & 0.35 & 8197 & 0.0228 & 0.73 & 0.27 \\
\hline$(\mathrm{M} 2)^{\mathrm{b}}$ & 106000 & 2.59 & 10500 & 0.05 & 0.34 & 10691 & 0.0318 & 0.72 & 0.28 \\
\hline$(\mathrm{M} 3)^{\mathrm{b}}$ & 77200 & 2.42 & 2900 & 0.013 & 0.36 & 2892 & 0.00938 & 0.76 & 0.24 \\
\hline$(\mathrm{M} 4)^{\mathrm{b}}$ & 66200 & 2.48 & 2450 & 0.010 & 0.33 & 2502 & 0.00728 & 0.75 & 0.25 \\
\hline$(\mathrm{M} 5)^{\mathrm{b}}$ & 89900 & 2.45 & 8140 & 0.0323 & 0.356 & 8304 & 0.0221 & 0.74 & 0.26 \\
\hline$(\mathrm{M} 6)^{\mathrm{b}}$ & 108000 & 2.14 & 7800 & 0.025 & 0.32 & 7787 & 0.0193 & 0.73 & 0.27 \\
\hline$(\mathrm{M} 7)^{\mathrm{b}}$ & 108000 & 1.86 & 10000 & 0.0398 & 0.338 & 10153 & 0.0283 & 0.748 & 0.252 \\
\hline$(\mathrm{L} 1)^{\mathrm{c}}$ & 92600 & 4.0 & 11200 & 0.158 & 0.453 & 10659 & 0.126 & 0.599 & 0.4 \\
\hline$(\mathrm{L} 2)^{\mathrm{c}}$ & 72400 & 3.58 & 3200 & 0.05 & 0.45 & 3808 & 0.0546 & 0.56 & 0.44 \\
\hline$(\mathrm{L} 3)^{\mathrm{c}}$ & 114000 & 4.21 & 25500 & 0.5 & 0.49 & 26510 & 0.583 & 0.51 & 0.49 \\
\hline$(\mathrm{L} 4)^{\mathrm{c}}$ & 82900 & 4.00 & 5900 & 0.1 & 0.47 & 5746 & 0.0825 & 0.57 & 0.43 \\
\hline
\end{tabular}

${ }^{a}$ Linear anionic polymerization followed by hydrogenation.

${ }^{\mathrm{b}}$ Commercial Metallocene gas phase polyethylenes.

${ }^{\mathrm{c}}$ Commercial Ziegler gas phase polyethylenes.

onto a single curve. The principal distinction from polymer to polymer within a given catalyst family is the average molecular weight; the shape of the MWD is approximately uniform within a given family. The time/ molecular weight shifting that collapses the spectra of different molecular weights onto a single curve illustrated in Fig. 2 is not a new concept. Indeed, this principle constitutes the physical basis underlying Tuminello's method of determining MWDs, (Tuminello 1986). The shape of the relaxation spectrum is determined by the shape of the MWD. The shape of the MWD is in turn

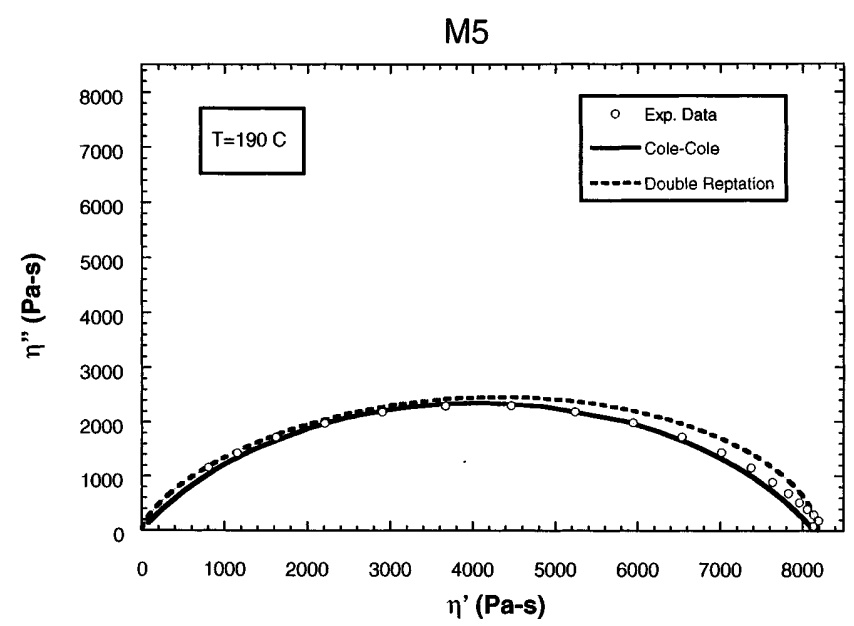

Fig. 1 Cole-Cole plot of a metallocene polyethylene resin, M5. The solid curve is the best fit to the Cole-Cole model and is typical of the quality of the fit that can be achieved controlled by the catalyst system and conditions used in the polymerization. Hence, each catalyst family has its own distinct relaxation spectra.

Figure 3 displays the non-dimensionalized model relaxation spectra versus dimensionless relaxation time (Marin et al. 1975) for the distinct catalyst families shown in 2. Here again, the spectra for each catalyst family collapses to its own unique curve. The modulus is scaled by the factor $\lambda_{0} / \eta_{0}$, which is approximately equal to the reciprocal plateau modulus, $G_{\mathrm{N}^{-1}}$. Hence, the scaled relaxation spectra are of order unity. In this format, the relationship between the relaxation spectrum and the underlying MWD is apparent. Specifically, narrow dimensionless distributions of relaxation times correspond to narrow MWDs. Conversely, broad MWDs yield broad relaxation spectra. Not surprisingly, dispersion in the MWD and dispersion in the relaxation spectrum go hand in hand. Quantitatively, Mead (1996) has shown how to directly relate the mechanical relaxation spectrum to the underlying MWD for the double reptation model. A similar relationship can be derived from the Cole-Cole model by using the relationship $\lambda=\mathrm{KM}^{3,4}$ to convert the time axis to a molecular weight axis and subsequently multiplying the relaxation modulus by a scale factor as described in Mead (1996). The net result of these manipulations is that the characteristic relaxation spectra yield characteristic MWDs associated with each catalyst family.

The half-width of the relaxation spectrum in Fig. 3 is directly related to $\alpha$ and is a useful measure of the processability of the melt. Specifically, the broader the 


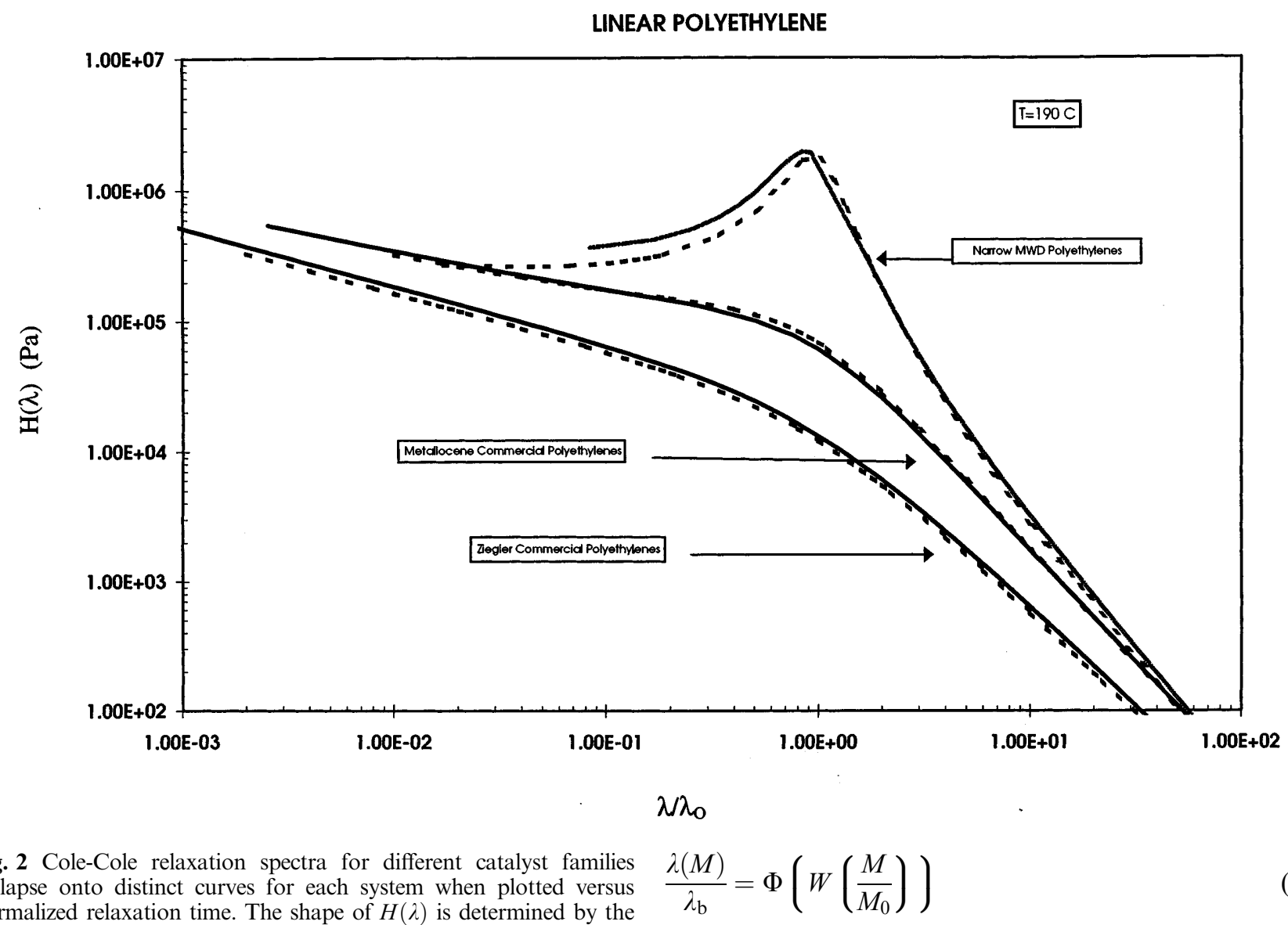
normalized relaxation time. The shape of $H(\lambda)$ is determined by the shape of the molecular weight distribution (MWD). Plotted are representative curves for the three polymer families: narrow MWD polyethylenes: solid line $(\mathrm{H})$, dashed line $(\mathrm{I})$; metallocene commercial polyethylenes: solid line (M1), dashed line (M4); Ziegler commercial polyethylenes: solid line (L2), dashed line (L4)

MWD, the greater the elastic character of the material. High melt elasticity is generally associated with susceptibility to flow instabilities (Mead 1994). Broad MWDs are also associated with an early onset of shear thinning character in the flow curve, a generally desirable property in extrusion operations. Thus, the correspondence of the MWD with the relaxation spectrum allows for ready extrapolation of the linear viscoelasticity data to more general characterizations concerning the processability of the material.

The collapse of the relaxation spectra of separate catalyst families onto distinct curves leads us to seek a relatively simple dependence of the shape of the relaxation spectrum on the shape of the MWD. Analytically, the families of unique curves in Figs. 2 and 3 suggests that the relaxation spectrum can be written as;

$H\left(\frac{\lambda}{\lambda_{0}}\right)=\phi\left[W\left(\frac{M}{M_{0}}\right)\right]$

Here, $W(x)$ is the weight-based MWD and $M_{0}$ is a parameter that scales the absolute molecular weight while preserving the functional shape of the normalized spectrum for a given family of polymers. Physically, Eq. (3a) represents the idea that the normalized relaxation spectrum is a fingerprint of the shape of the MWD. Equation (3b) describes the relaxation time of a component of the MWD. The relaxation time of the component depends on its absolute molecular weight and the details of the MWD. Thus, the relaxation spectrum is a complex functional of the normalized MWD function, $W\left(M / M_{0}\right)$. This dependence is consistent with Fig. 3, which clearly illustrates dependence of the normalized relaxation spectra on the shape of the MWD. More fundamentally, Eq. (3b) suggests that time and molecular weight are transformable, an observation that has been used with success in the determination of molecular weights from linear viscoelasticity data (Mead 1994; Tuminello 1986; Wasserman 1995).

An analytic expression for the relaxation spectrum $H(\lambda)$ of the Cole-Cole model can be derived from Eq. (2). The details of the analysis can be found in Gross (1953) (Labaig et al. 1973; Lanfray 1988), the result is: 


\section{LINEAR POLYETHYLENE}

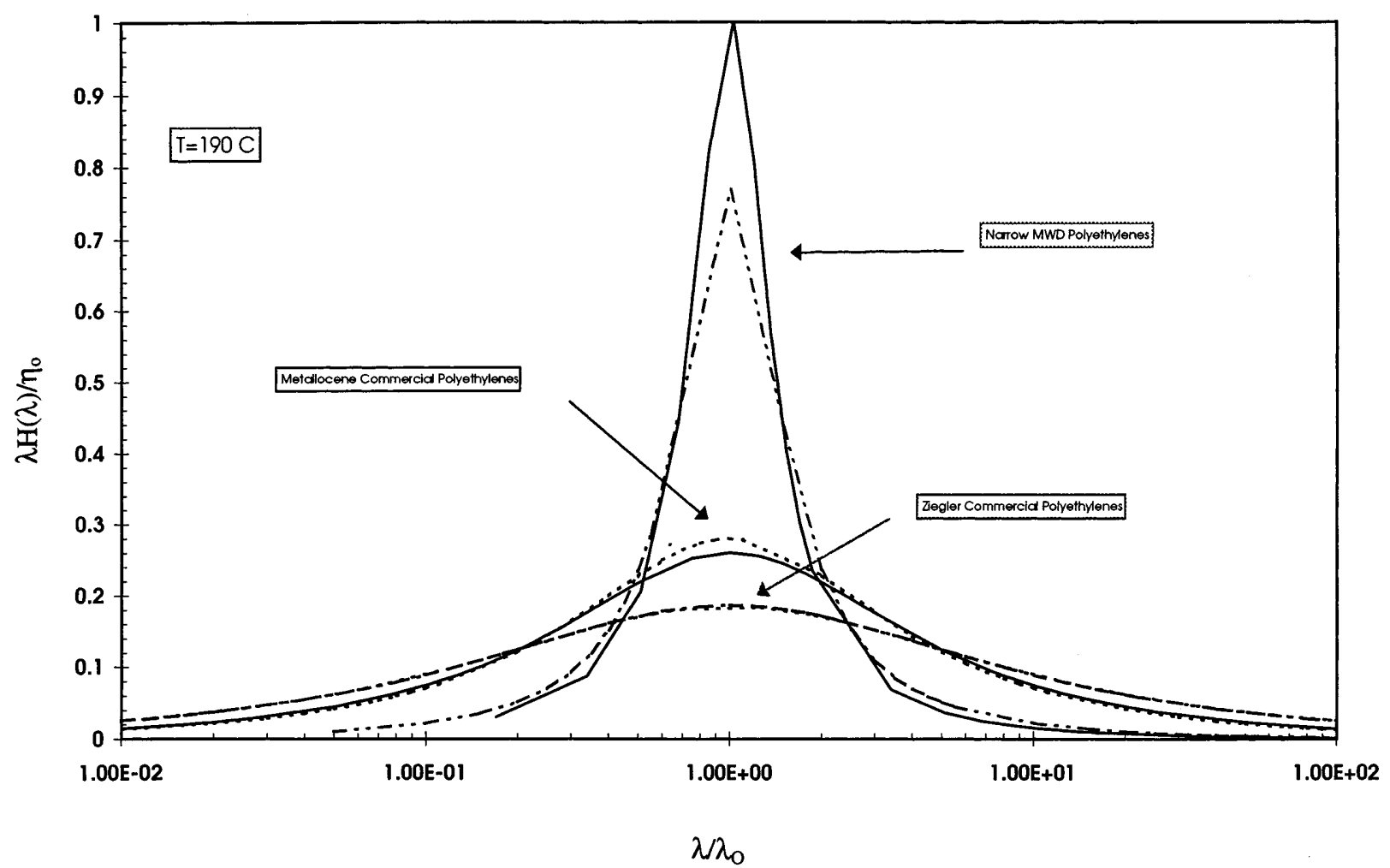

Fig. 3 Dimensionless plot of the relaxation spectra of different catalyst systems corresponding to Fig. 2. Dispersion in the relaxation modulus and dispersion in the MWD go hand in hand

$H(\lambda)=\frac{\eta_{0}\left(\frac{\lambda_{0}}{\lambda}\right) \sin (\alpha \pi)}{\pi \lambda\left[1+\left(\frac{\lambda_{0}}{\lambda}\right)^{2(1-\alpha)}-2\left(\frac{\lambda_{0}}{\lambda}\right)^{(1-\alpha)} \cos (\pi \alpha)\right]}$

If the zero shear viscosity parameter $\eta_{0}$ is identified with $G_{\mathrm{N}} \lambda_{0}$, as is typically done, Eq. (4) is a function of $\lambda / \lambda_{0}$ and $\alpha$ only. Furthermore, if the parameter $\alpha$ is a function of the shape of the MWD only, we can anticipate the collapse of families of polymers onto a single curve from the Cole-Cole model when the relaxation time is normalized by $\lambda_{0}$. These tenets are consistent with Eq. (3) and the experimental data in Figs. 2 and 3.

Figure 4 is a linear-linear plot of the relaxation spectrum. The linear frequency scale reveals features of the short time scale behavior in a transparent manner. From Eq. (4) a singularity at $\lambda=0$ is evident, specifically for small relaxation times, $H(\lambda) \sim \lambda^{-\alpha}$. According to the Bueche-Ferry theorem, the behavior of the model relaxation spectrum at short time scales is of no consequence with respect to molecular characterization. Specifically, the Bueche-Ferry theorem states that the short time scale behavior of all polymers of a given chemical composition is identical, independent of mo-

lecular weight (Doi 1987). Physically, this result is a manifestation of the fact that short time scale phenomena are associated with motions of small portions of the chain. Unless a short piece of chain resides close to a chain end, its dynamics are unaffected by the absolute magnitude of molecular weight of the polymer within which it resides. Therefore, as a molecular weight characterization tool, only the longer time scale plateau and terminal relaxation modes corresponding to relax-

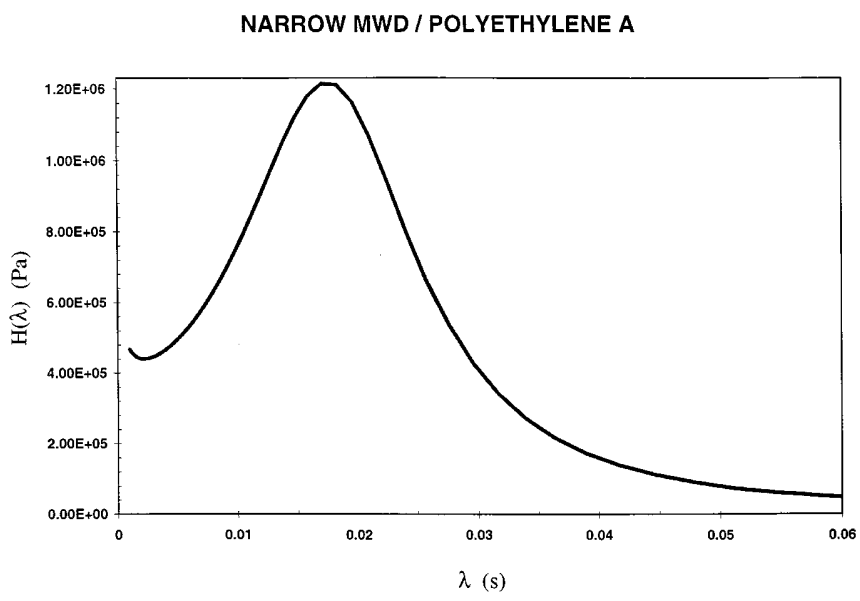

Fig. 4 Linear plot of the best fit Cole-Cole model relaxation spectrum for sample A. The singular behavior at $\lambda=0$ is evident 
Fig. 5 Cole-Cole relaxation time $\lambda_{0}$ versus $M_{\mathrm{w}}$. Distinct power law relationships exist for each catalyst family. All slopes are approximately equal

\section{LINEAR POLYETHYLENE}

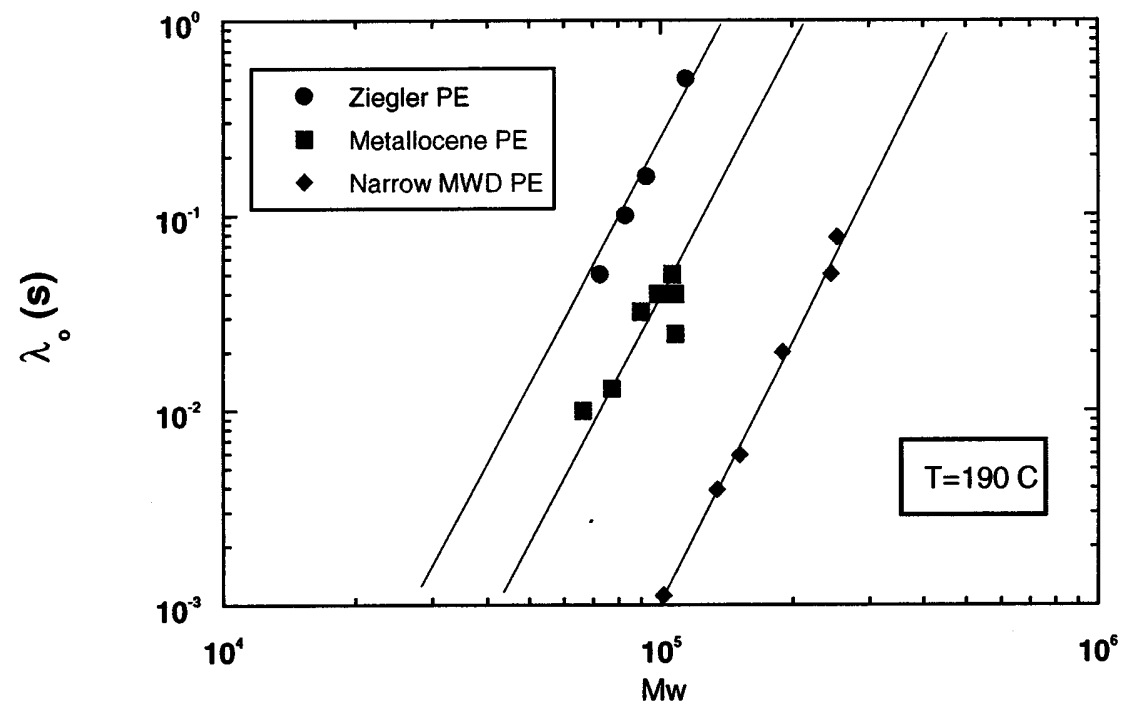

ation of the entire chain are relevant. The terminal and plateau regions are precisely where the Cole-Cole model is accurate and viable.

The parameters of the Cole-Cole distribution for the families of polymers are cataloged in Table 1. A systematic variation of the parameters with moments of the MWD is evident. Given the correspondence between the Cole-Cole relaxation spectrum and the double reptation model illustrated in Fig. 1, this is not entirely surprising. In Fig. 5 through 9, we plot the ColeCole fitting parameters versus various combinations of moments of the MWD in order to establish the relationship, if any, between the two. Figure 5 displays the Cole-Cole relaxation time $\lambda_{0}$ versus $M_{\mathrm{w}}$. As with the relaxation spectrum, a distinct curve emerges for each catalyst family. The curves are nearly linear, suggesting a power law relationship for each family. The dependence of $\lambda_{0}$ on moments of the MWD can be simplified further by plotting against $M_{\mathrm{w}}\left(M_{\mathrm{w}} / M_{\mathrm{n}}\right)$ as shown in Fig. 6. All lines collapse onto a single universal power law relationship for polyethylene systems of the form,

$\lambda \propto\left[M_{\mathrm{w}}\left(\frac{M_{\mathrm{w}}}{M_{\mathrm{n}}}\right)\right]^{3.9}$
Fig. 6 Cole-Cole relaxation time $\lambda_{0}$ versus $M_{\mathrm{w}}\left(M_{\mathrm{w}} / M_{\mathrm{n}}\right)$. A power law relationship exists for all polyethylene resins studied in this work

\section{LINEAR POLYETHYLENE}

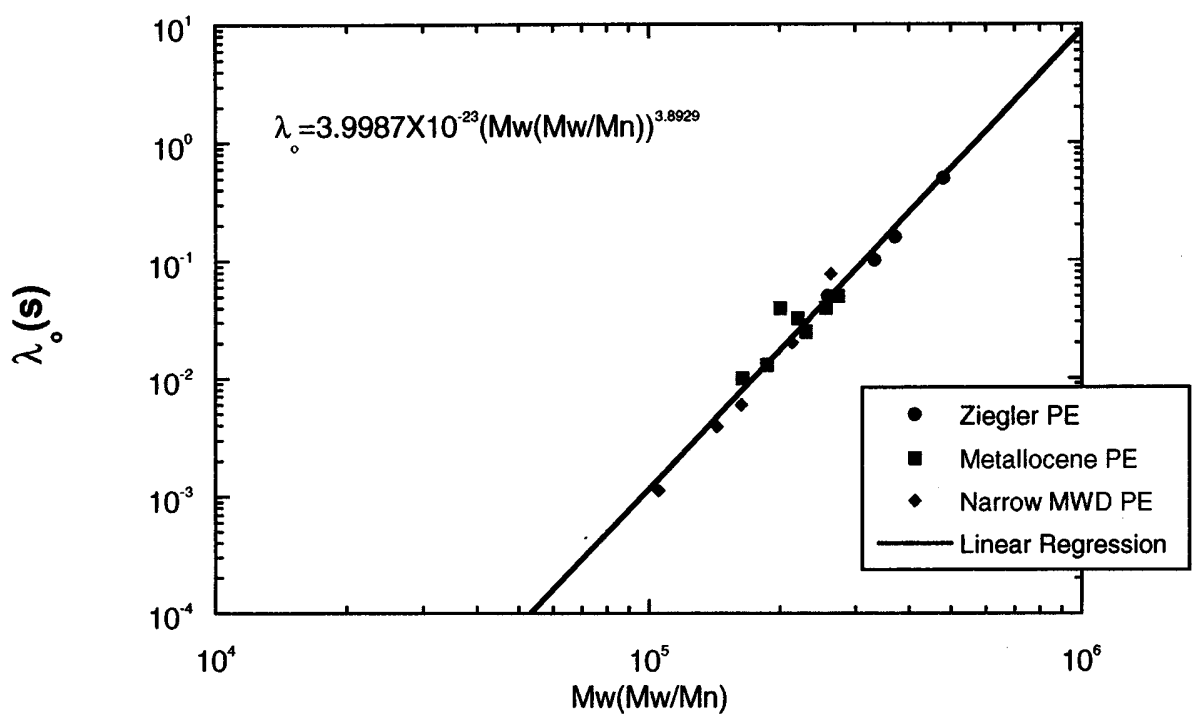


Fig. 7 Cole-Cole zero shear viscosity versus $M_{\mathrm{w}}\left(M_{\mathrm{w}} / M_{\mathrm{n}}\right)$. Each catalyst family has a distinct correlation with virtually identical slopes shifted horizontally

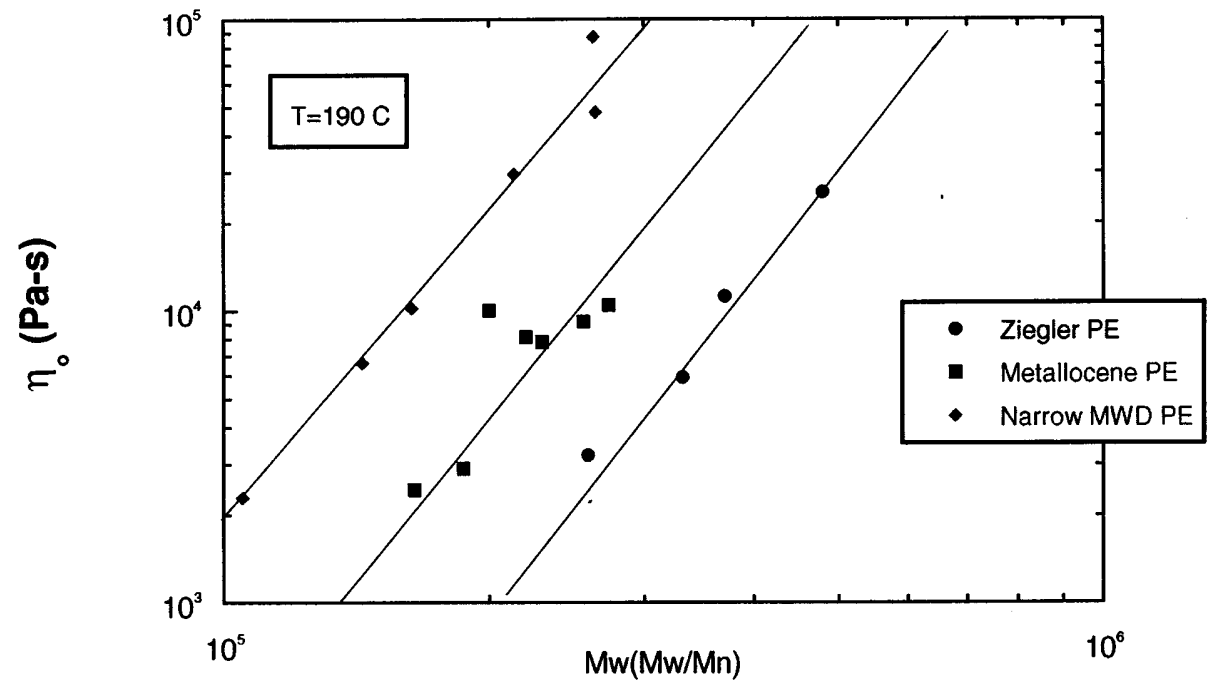

The empirical dependence of the characteristic relaxation time on moments of the MWD is similar to that found for the so-called double reptation mixing rule.

A more widely studied material property is the zero shear viscosity, $\eta_{0}$. Figure 7 shows a log-log plot of $\eta_{0}$ versus $M_{\mathrm{w}}\left(M_{\mathrm{w}} / M_{\mathrm{n}}\right)$. Once again, a distinct power law relationship emerges for each individual catalyst family. Each family has approximately the same slope, but they are slightly offset from each other.

$\eta_{0} \propto\left[M_{\mathrm{w}}\left(\frac{M_{\mathrm{w}}}{M_{\mathrm{n}}}\right)\right]^{3.4}$

Each polymer family is synthesized from a different catalyst system under homogeneous reaction conditions. Polymers derived from the same catalyst system all have MWDs with similar shapes but differing average molecular weights. The shape of the MWD is a characteristic signature of a specific catalyst system.

The molecular weight dependence of the zero shear viscosity can be collapsed into a universal curve by independently manipulating the weight average molecular weight and the polydispersity index, $M_{\mathrm{w}} / M_{\mathrm{n}}$, factors. Figure 8 illustrates the results of this exercise. A universal power law relationship emerges for the zero shear viscosity of the polyethylene resins studied.

$\eta_{0} \propto\left[M_{\mathrm{w}}\left(\frac{M_{\mathrm{w}}}{M_{\mathrm{n}}}\right)^{0.3}\right]^{3.3}$

The dependence of the zero shear viscosity on catalyst parentage is an unambiguous signature of sensitivity to the detailed shape of the MWD. The breadth of the MWD, as quantified by $M_{\mathrm{w}} / M_{\mathrm{n}}$, is one relatively simple means to characterize the shape of the MWD. A more detailed description would require knowledge of additional moments of the MWD. It is easy to show, using Fourier transforms, that knowledge of all moments of the MWD is equivalent to full knowledge of the MWD function.

The above scaling relationship for the zero shear viscosity is similar to that reported by Locati and Gargani (1973), Wasserman (1994), and Zeichner and Patel (1981). However, our study is the first to clearly illustrate the distinct catalyst family/polymerization conditions dependence of the zero shear viscosity as well as its correspondence to measures of dispersion in the MWD. As Eq. (7) suggests, the zero shear viscosity is most sensitive to weight average molecular weight with a weaker dependence on the shape of the MWD, particularly the high molecular weight tail.

The $\alpha$ parameter is approximately constant within a given family of polymers indicating that $\alpha$ is a function of the dispersion in the MWD and not the absolute molecular weight. Figure 9 illustrates the dependence of $\alpha$ on one measure of the dispersion in the MWD, $M_{\mathrm{w}} / M_{\mathrm{n}}$. Although no simple power law relationship exists, there is a definite correlation of $\alpha$ and $M_{\mathrm{w}} / M_{\mathrm{n}}$ for the various systems studied since polymers from all families collapse onto a single curve, as expected from the discussion following Eq. (3).

Summarizing, we have established simple empirical power law relationships between some Cole-Cole model parameters and moments of the underlying MWD. These relationships can be used to rapidly and reliably determine the shape of the MWD from experimental linear viscoelasticity data. In this respect, the Cole-Cole model is an extremely powerful yet simple characterization methodology. In the next section we demonstrate that the Cole-Cole parameters are related to the Cross 
Fig. 8 Cole-Cole zero shear viscosity versus $M_{\mathrm{w}}\left(M_{\mathrm{w}} / M_{\mathrm{n}}\right)^{0.3}$. All catalyst families collapse to the same universal correlation

\section{LINEAR POLYETHYLENE}

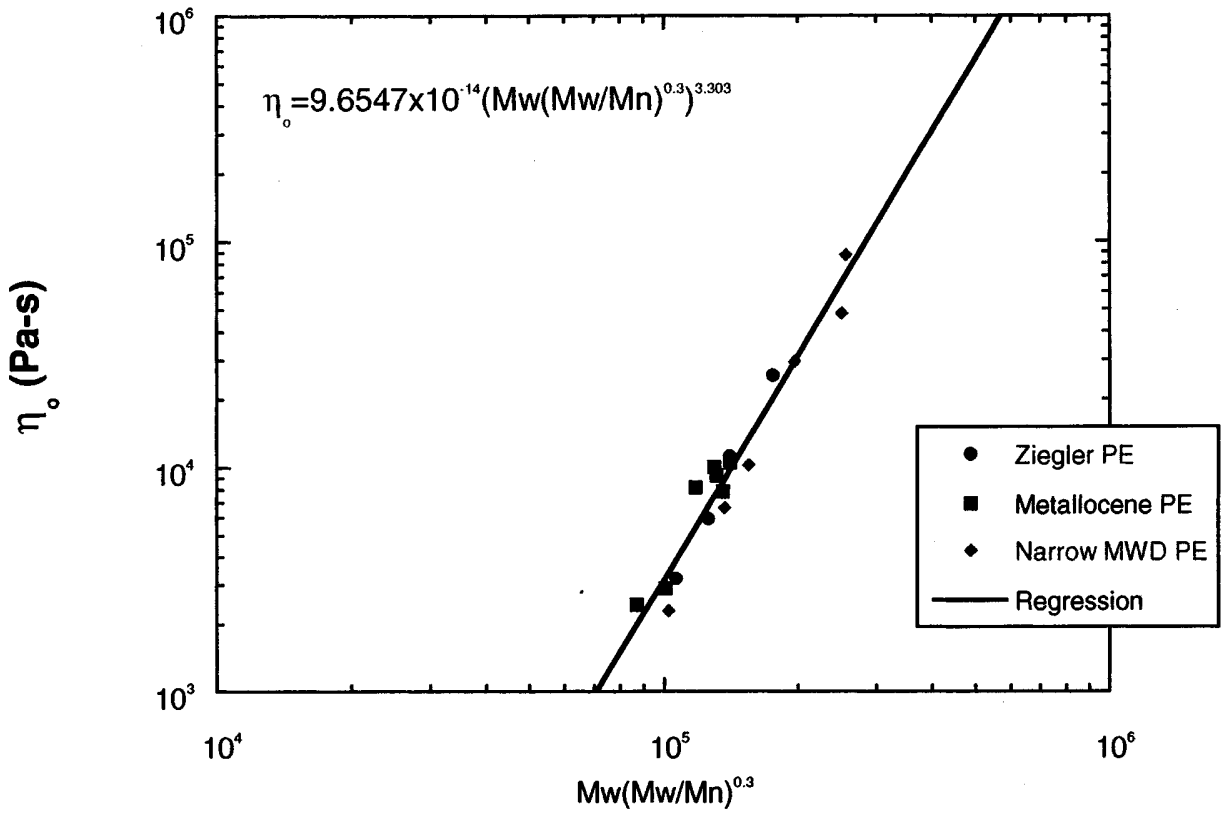

equation parameters. Hence, the empirical relations developed for the relaxation spectrum parameters can be applied to the flow curve.

\section{Analysis of the relationship between the Cole-Cole distribution and the Cross model for the flow curve}

In this section we compare the parameters of the ColeCole model of the dynamic linear viscoelastic material properties and the Cross model for the flow curve and establish a direct correspondence between the two parameter sets. In the context of polymer processing, linear viscoelasticity itself is of little consequence. The

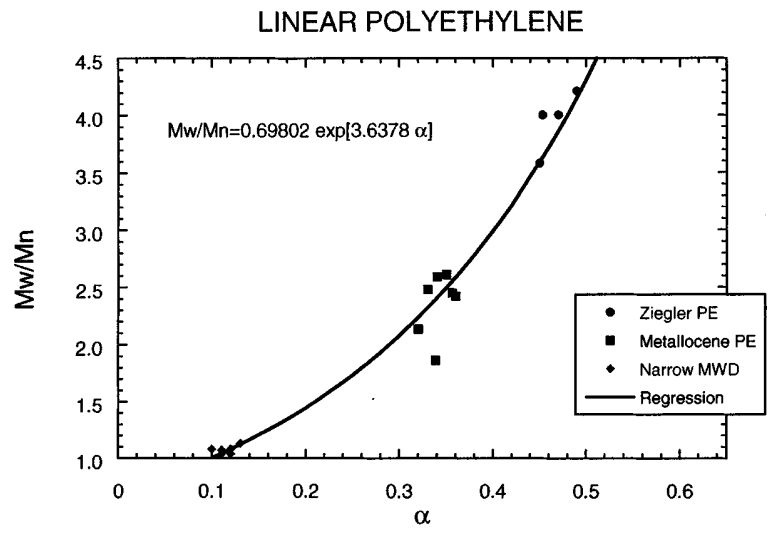

Fig. $9 M_{\mathrm{w}} / M_{\mathrm{n}}$ versus the Cole-Cole $\alpha$ parameter. A complex, nonpower law correlation exists for all the resins examined practical utility of the linear viscoelastic relaxation spectrum lies in the ability to use it to characterize more general, non-linear flow properties which are intrinsically related to the processability of a material. Although a detailed understanding of how the linear viscoelastic material properties are related to the nonlinear properties is currently not fully understood, there exist many well established empirical correlations linking the two. For example, many systems of practical interest, such as polydisperse linear or branched flexible polymers, obey the empirical Cox-Merz rule (Cox and Merz 1958).

$\eta(\gamma)=\left|\eta^{*}(i \omega)\right|_{\gamma=\omega}$

This powerful relationship allows the non-linear steady flow properties to be determined from linear viscoelastic measurements alone (Mead and Leal 1995; Yasuda et al. 1981). The ability to predict the non-linear flow properties directly from linear viscoelasticity data is a profound as well as practical capability that requires further discussion. As a practical matter, the applicability of the Cox-Merz rule allows the flow curve to be approximately determined from dynamic viscometry, which complements capillary rheometry by extending the accessible shear rate range.

Until recently, the fundamental physics underlying the Cox-Merz rule have remained an enigma. However, Marrucci recently published an analysis offering a plausible physical explanation for the Cox-Merz rule (Marrucci 1996). Briefly, the Cox-Merz rule is observed because in large deformation flows, a parallel convective relaxation process with a rate proportional to the 
deformation rate becomes increasingly important. This deformation rate dependent convective relaxation mechanism competes with the diffusive mechanism and systematically eliminates contributions from the long time scale diffusive relaxation processes. In this simplified manner, the physical mechanism underlying the Cox-Merz rule can be understood.

When the Cox-Merz rule is applicable, one can directly calculate the flow curve from experimental linear viscoelasticity data. The flow curve is a critical property in extrusion operations since it controls the pressure level developed at the die head. In particular, the degree of shear thinning a material exhibits is of particular practical importance since most processing operations run at high throughputs.

The Cross model for the flow curve is an empirical model with three parameters (Cross 1969), $\eta_{0 \mathrm{c}}, \tau$ and $\mathrm{m}$ :

$\eta(\gamma)=\frac{\eta_{0 \mathrm{c}}}{1+(\tau \gamma)^{m}}$

Both $\eta_{0 c}$ and $\tau$ depend on temperature in the usual Arrhenius-like manner. The parameter $m$ is independent of temperature. A three-parameter model can only capture the three most salient features of the flow curve. The three features of the flow curve the Cross model captures are (see Fig. 10);

1. The zero shear plateau, $\eta_{0 \mathrm{c}}$

2. The characteristic shear rate at which shear thinning commences, $\gamma \sim 1 / \tau$

3 . The slope of the high shear rate portion of the curve, $m$

Flow curves with more complex features cannot be modeled with the Cross equation. Additionally, the details of the transition from linear behavior to shear thinning behavior is pre-determined by the functional form of (9). More complex transitional behaviors are possible and models such as those of Carreau (1969), Bueche-Harding (1958) and Yasuda et al. (1981) have been developed to account for such situations.

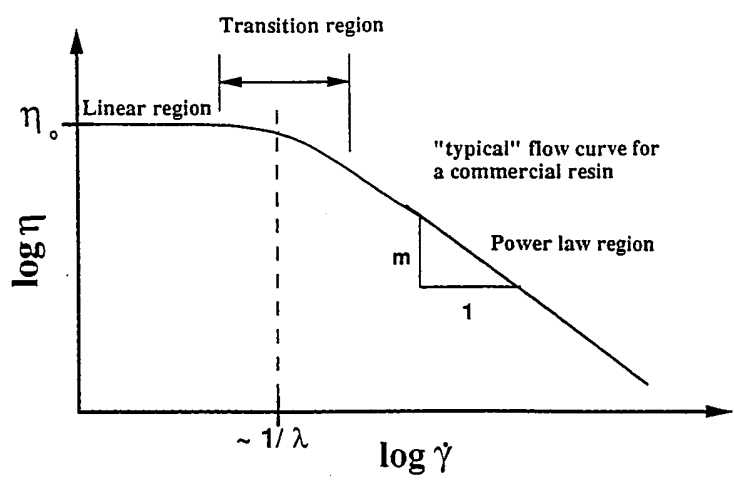

Fig. 10 Sketch of the three principal features of the flow curve for a material that obeys the Cross equation
The Cox-Merz rule (8) was applied to experimental linear viscoelasticity data for the polyethylene systems in Table 1 to determine the flow curve. The parameters of the Cross model for these data were determined via a non-linear regression method that yielded excellent fits to the flow curve. The Cross model parameters are reported in Table 1.

Examining the limiting cases of high and low $\omega$ in applying Eq. (8) to (2) shows that the Cole-Cole parameters map directly onto the Cross model parameters when the Cox-Merz rule applies. Specifically, both $\eta_{0 \mathrm{c}}$ and $\tau$ translate directly from one model to the other. The final parameter $m$ is equal to $1-\alpha$. Since the ColeCole parameters are directly related to the Cross equation parameters when the Cox-Merz rule holds, the empirical relations relating the Cole-Cole parameters to moments of the MWD can in principle be directly applied to the Cross model parameters (see Figs. 5-9). In this manner, the flow curve can also be used to determine the moments of the MWD when the CoxMerz rule applies quantitatively. In this sense, linear viscoelastic methods of determining the MWD are equivalent to the flow curve inversion method which is an intrinsically non-linear flow situation (Malkin and Teishev 1991; Shaw and Tuminello 1994; Wood-Adams and Dealy 1996) Although the two methods are equivalent in principle, their relative sensitivities with respect to determining the MWDs are quite different in practice. Specifically, it is often difficult to unambiguously determine the parameters $\eta_{0 \mathrm{c}}$ and $\tau$ directly from flow curve data.

Difficulties related to the finite range of the experimental data sets are reflected in the approximate correspondence between the Cross and Cole-Cole model parameters in Table 1. The correspondence is particularly poor for the narrow distribution polymers. Specifically, the slope of the flow curve for several of the narrow distribution polymers is determined to be less than -1 , which is theoretically impossible since the stress must be a monotonically increasing function of the shear rate (Doi and Edwards 1986). The reason for this pathological result is that the narrow distribution polymers are so nearly Newtonian in character that the parameter determination routine can choose a range of values of $m$ without seriously deteriorating the quality of the fit. Similar effects occur to the other polymer systems to a lesser extent since a greater portion of the non-linear portion of the flow curve is available to fit. These points are illustrated graphically in Fig. 11 where the flow curve of one representative member of each catalyst family is plotted. In addition to these difficulties, it has also been noted that the Cox-Merz rule improves with increasing breadth of the MWD (Larson 1985). The correspondence of the Cole-Cole model to the Cross model is predicated on the applicability of the CoxMerz rule and any deterioration of the veracity of this 
Fig. 11 Flow curves calculated by applying the Cox-Merz rule, Eq. (8), to the dynamic mechanical data for representative members of the catalyst families of this study. The narrow distribution polymers do not possess sufficient non-linear behavior to allow a sensible determination of the $m$ parameter

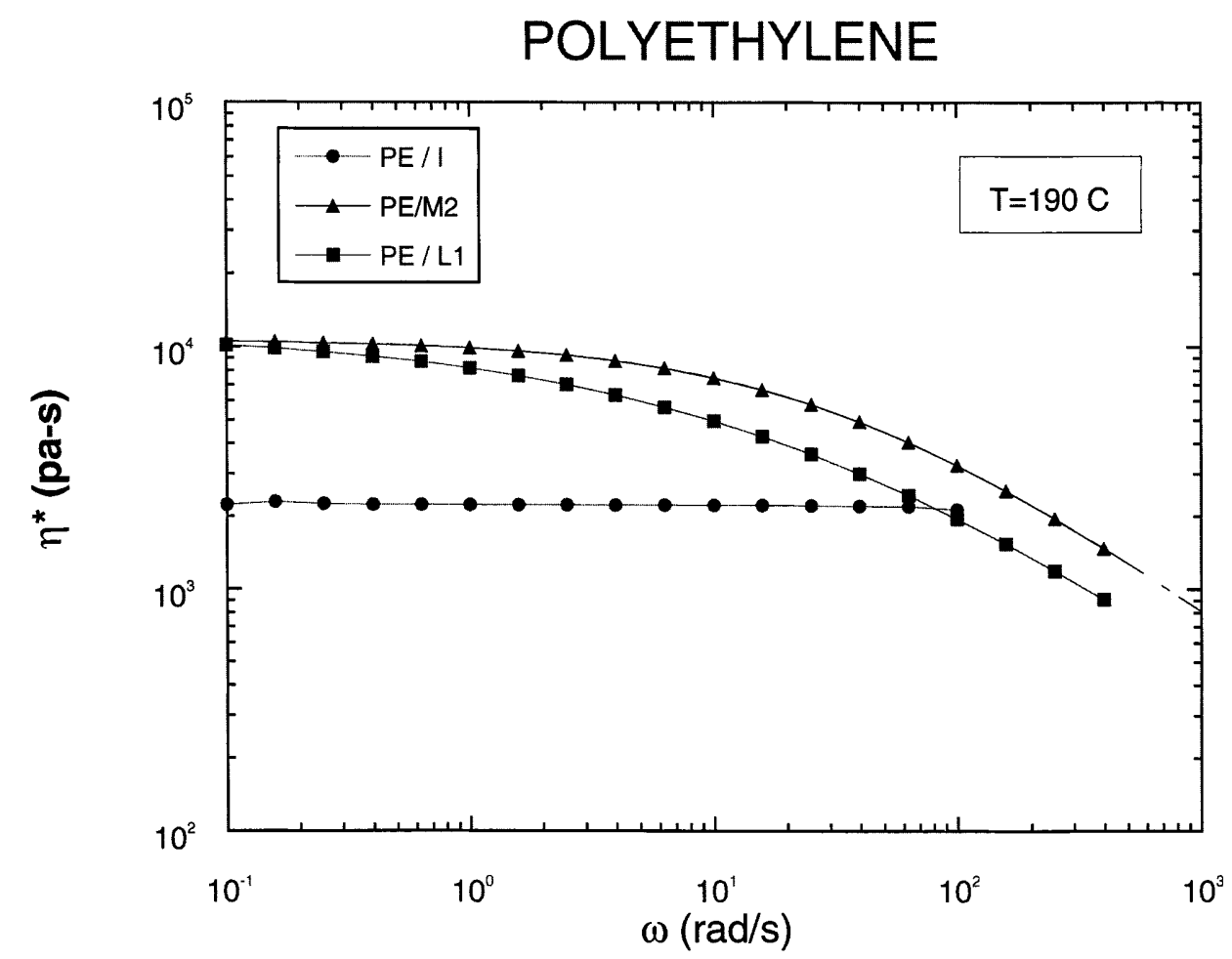

empirical correlation will be reflected in the fitting parameters of the Cross equation. Our calculations are consistent with these notions. With the previous provisos, the correspondence between the Cole-Cole model and the Cross model parameters is quite compelling.

With the parameter identification detailed above and the correlations of model parameters with molecular parameters, we can interpret qualities of the MWD and their impact on the flow curve. Specifically, the Cross parameter $m$ is equal to $1-\alpha$, which is a function of the dispersion in the MWD (Fig. 9). Low polydispersity generates a small value of $\alpha$ and hence and large slope for the flow curve. This prediction is qualitatively consistent with experimental observation (Dealy and Wissbrun 1990). Conversely, broad MWDs have larger $\alpha$ values (Table 1) and hence enhanced shear thinning behavior. The characteristic time parameter $\lambda$ has a strong dependence on the weight average molecular weight with a weaker dependence on the dispersion. Here again, these dependencies are consistent with empirical observations for the flow curves of polydisperse systems (Figs. 5, 6).

\section{Discussion}

The usefulness of the Cole-Cole model relaxation spectrum lies principally in its ability to accurately capture the long time scale terminal behavior of monomodal linear flexible polymers with a relatively small number of empirical parameters that can be readily interpreted. The parametric dependence of Cole-Cole plots on the frequency brings out the plateau and terminal behavior in a particularly obvious manner. The short time scale behavior, which is of no value with respect to polymer characterization, is effectively muted in this representation. However, the Cole-Cole distribution is a continuous distribution defined over the entire relaxation time axis. Therefore, along with the desired representation of the long time scale phenomena, a short time scale response associated with "glassy" modes is also inferred. However, our procedure for determining the Cole-Cole parameters described in the appendix demonstrates that the short time scale phenomena does not enter into the parameter determination and hence the material characterization.

The criteria for success of a material characterization protocol depends on the information required and the viability of the method. While there exist elaborate theories describing the molecular dynamics of polydisperse systems of linear flexible polymers, they are frequently so complex mathematically that they are of little practical value in characterizing commercial polymers. For example, the models of Viovy et al. (1991) and Watanabe and Kotaka (1987 a, b) provide a detailed, elaborate description of the dynamics of polydisperse systems. However, the ability to apply these models to characterize any system beyond a model bidisperse blend would require a prohibitive amount of computational effort. The "double reptation" model provides a 
mathematically viable approximation to Viovy et al. as well as Watanabe and Kotaka that is analytically tractable. The existence of the more rigorous model allows quantitative estimates to be made of when the double reptation model fails (Milner 1996). The ColeCole model is a step further removed from rigorous molecular models than "double reptation" with a commensurate reduction in mathematical complexity.

In any model there are varying degrees of rigor which depend on the level of detail included in the model. Generally, the more detailed the model is, the more mathematically untractable it becomes. However, if a model is overly simplified it may omit key physical features of the problem. Thus, constructing models is a delicate balance between realism and the practical need for mathematical simplicity. The above discussion reveals that there exists a hierarchy of theoretical rigor for models of the mechanical behavior of polydisperse systems of linear flexible polymer. Which model is most appropriate to use depends on the specific application. Although the fit of experimental data to the Cole-Cole model is typically very good, a detailed physical or molecular interpretation of this observation is not readily apparent since the Cole-Cole relaxation spectrum is empirical. The existence of rigorous detailed theories is important in this regard. Additionally, since it is impossible to extrapolate simple empiricism to new situations, access to rigorous theoretical models is extremely valuable.

The principal attraction of the Cole-Cole polymer characterization methods described in this paper lie in the relative simplicity of the experimentation and analysis coupled with the fundamental nature of the molecular and rheological information gleaned. In particular, the power law correlations of the Cole-Cole model parameters with moments of the MWD provide powerful and rapid assessments of the salient features of the MWD. Apart from the specific chemical structure, the MWD is the most fundamental material character- istic of a system of linear flexible polymers. In principle, from the characteristics of the MWD, all mechanical properties of the melt, linear and non-linear, can be deduced within scale factors (Doi and Edwards 1986). Given the mathematical complexity associated with virtually every theoretically derived structure-property relationship (Doi and Edwards 1986; Milner 1996), the accuracy and simplicity of the empirical Cole-Cole characterization methodology is truly remarkable. The Cole-Cole model relaxation spectrum provides a characterization technique that is experimentally as routine as an MFI yet has vastly greater discrimination and resolution capabilities for systems of linear flexible polymer. Physically, the enhanced capabilities result from the incorporation of a more complete description of the complex time dependence of the material.

Despite the fact that many systems of commercial polymer do obey the Cole-Cole type distribution, there are several systems that do not. In particular, some topologically complex systems, such as branched flexible polymer, require the four parameter Havriliak-Negami model relaxation spectrum to accurately capture the linear viscoelastic relaxation spectrum (Havriliak and Negami 1967). The Cole-Cole model is a special case of the Havriliak-Negami distribution. We should note that in a manner similar in which the Cole-Cole model corresponds to the Cross model, the Havriliak-Negami model relaxation spectra corresponds to the Carreau flow curve model. Application of other model relaxation spectra to more complex branched polymer systems is an obvious way to further build on the ideas presented in this paper.

Acknowledgements The authors would like to express their gratitude to Exxon Chemical Company for permission to publish this work. The authors would also like to thank Drs. S. C. Speed, and S. T. Milner, for valuable technical discussions, Dr. L. Fetters for synthesizing the anionic polyethylenes, Mr. A. N. Jared, and Dr. R. Mendelson, for the rheological measurements of the commercial and anionic polyethylenes, respectively.

\section{References}

Bremner T, Rudin A, Cook DG (1990) Melt flow index values and molecular weight distributions of commercial thermoplastics. J Appl Polym Sci 41:1617

Bueche F, Harding SW (1958) A new absolute molecular weight method for linear polymers. J Polym Sci 32:177

Burke JJ, Weiss V (eds.) (1975) Characterization of materials in research: ceramics and polymers. Syracuse University Press Syracuse

Carreau PJ (1969) Ph. D. thesis, University of Wisconsin
Carrique F, Delgado AV (1996) Dielectric relaxation pattern of dilute colloidal suspensions. Colloid Polym Sci 274:848-853

Cole KS, Cole S (1941) Dispersion and absorption in dielectrics. J Chem Phys 9:341

Cox WP, Merz EH (1958) Correlation of dynamic and steady flow viscosities. J Polym Sci 28:619

Cross MM (1969) Polymer rheology: influence of molecular weight and polydispersity. J Appl Polym Sci 13:765
Dealy JM, Wissbrun KF (1990) Melt rheology and its role in plastics processing. Van Nostrand, New York

des Cloizeaux J (1988) Double reptation vs. simple reptation in polymer melts. J Europhys Lett 5:437-442

Doi M (1987) Basic principle in the viscoelasticity of polymeric liquids. JNNFM 23:151

Doi M, Edwards SF (1986) The theory of polymer dynamics. Oxford University Press

Fodor JS, Hill DA (1994) Determination of molecular weight distribution of 
entangled cispolyisoprene melts by inversion of normal-mode dielectric loss spectra. J Phys Chem 98:7674

Graessley WW (1982) Entangled linear, branched and network polymer systems-molecular theories. Adv Polym Sci 47:67

Gross B (1953) Mathematical structure of the theories of viscoelasticity. Hermann, Paris

Havriliak S, Negami S (1967) A complex plane representation of dielectric and mechanical relaxation processes in some polymers. Polymer 8:161 (1967)

Havriliak S Jr, Havriliak S (1993) ColeCole plots: methods for unbiased parameter estimation. J Mol Liq 56:49

Havriliak S Jr, Havriliak S (1994) Results from an unbiased analysis of nearly 100 sets of relaxation data. J Non-Cryst Solids 172-174:297-310

Huang J, Rempel GL (1995) Ziegler-Natta catalyst of olefin polymerization: mechanistic insights from metallocene systems. Prog Polym Sci 20:459-526

Labaig JJ, Monge Ph, Bednarik J (1973) Steady flow and dynamic viscoelastic properties of branched polyethylene. Polymer 14:384

Lanfray Y (1988) Thesis, Docteur de $3^{\mathrm{e}}$ cycle

Larson RG (1985) Constitutive relationships for polymeric materials with power-law distributions of relaxation times. Rheol Acta 30:327

Locati G, Gargani L (1973) Dependence of zero-shear viscosity on molecular weight distribution. J Polym Sci; Polym Lett Ed 11:95

Malkin AY, Teishev AE (1991) Flow curve - Molecular weight distribution: Is the solution of the inverse problem possible. Polym Eng Sci 31:1590

Marin G, Labaig JJ, P Monge P (1975) Dynamic viscoelasticity of entangled polymers. Polymer 16:223

Marrucci G (1996) Dynamics of entanglements: a nonlinear model consistent with the Cox-Merz rule. J Non-Newtonian Fluid Mech 62:279

McCall DW (1969) Molecular dynamics and structure of solids. Carter RS, Rush JJ (eds) National Bureau of Standards Special publication 301

Mead DW (1994) Determination of molecular weight distributions of linear flexible polymers from linear viscoelastic material functions. J. Rheol 38:1797

Mead DW (1996) Component predictions and the relaxation spectrum of the double reptation mixing rule for polydisperse linear flexible polymers. J. Rheol 40:633

Mead DW, Leal LG (1995) The reptation model with segmental stretch. Rheol Acta 34:339-359

Milner ST (1996) Relating the shear thinning curve to the molecular weight distribution in linear polymer melts. J. Rheol 40:303

Montfor JP (1976) Rheological behaviour of blends of narrow molecular weight distribution polystyrenes in the molten state. Dynamic viscoelastic properties in the terminal zone. Polymer 17:1054

Montfort JP, Marin G, Monge P (1984) Effects of constraint release on the dynamics entangled linear polymer melts. Macromolecules 17:1537

Nguyen TQ, Yu G, Kausch HH (1996) Some emerging techniques in polymer molecular weight distribution (MWD) characterization. Macromol Symp 110:97-106

Shaw MT, and Tuminello WH (1994) A closer look at the MWD-viscosity transform. Polym Sci. Eng. 34:159

Shenoy AV, Sani DR, Nadkarni VM (1984) Melt rheology of polymer blends from melt flow index Int Polym Mater 10:213-235

Taylor CR, Aloisio CJ, Matsuoka S (1985) Mechanical relaxation of a flame retardant polycarbonate using the ColeCole method. Polym Eng Sci 25:105
Thayer AM (1995) Metallocene catalysts initiate new era in polymer synthesis. C\& E News 73:15

Tsenoglou C (1987) Molecular weight polydispersity effects on the viscoelasticity of entangled linear polymers. ACS Polym Prepr 28:185

Tsenoglou C (1991) Molecular weight polydispersity effects on the viscoelasticity of entangled linear polymers. Macromolecules 24:1762- 1767

Tuminello WH (1986) Molecular weight and molecular weight distribution from dynamic measurements of polymers melts. Polym eng Sci 26:1339

Viovy JL, Rubinstein M, Colby RH (1991) Constraint relase in polymer melts: Tube reorganization versus tube dilation. Macromolecules 24:3587-3596

Wasserman S (1995) Calculating the molecular weight distribution from linear viscoelastic response of polymer melts. J Rheol 39:601

Wasserman SH (1994) The relationship between polydispersity and linear viscoelasticity in entangled polymer melts. Ph.D. thesis, Princeton University

Watanabe H, Kotaka T (1987) Viscoelastic and diffusion properties of binary blends of monodisperse polystyrenes. Macromolecules 20:530-535

Watanabe H, Kotaka (1987) Entanglements in binary and ternary blends of narrow molecular weight distribution polystyrenes. Macromolecules 2:535-543

Wood-Adams PM, Dealy JM (1996) Use of rheological measurements to estimate the molecular weight distribution of linear polyethylene. J Rheol 40:761

Yasuda KY, Armstrong RC, Cohen RE (1981) Shear flow properties of concentrated solutions of linear and star branched polystyrenes. Rheol Acta 20:163

Zeichner GR, Patel PD (1982) The influence of molecular weight distribution in melt rheology of polypropylene. J Rheol 26:93

\section{Appendix: Experimental determination of the Cole-Cole model parameters}

In order to fully utilize the Cole-Cole model to characterize systems of linear flexible polymer, a viable procedure for determining the model parameters must be established. Methods for unbiased statistical parameter estimation in the context of the Cole-Cole dielectric relaxation spectrum have previously been reported by Havriliak and Havriliak (1993, 1994). These procedures yield an optimal fit to the experimental data in a statistical sense by minimizing a prescribed error norm.

Our methods for determining the mechanical relaxation spectrum Cole-cole parameters are not statistically optimized in the above sense. The parameter determination methods described in this appendix are based on identifying the principal salient features of the mechanical relaxation spectrum and subsequently using this discrete set of features to determine the empirical parameters. In this sense, we do not use all of the information contained in the relaxation spectrum but rather a discrete subset of it. However, for mechanical relaxation spectra of linear flexible polymers with a monomodal molecular weight distribution, the relaxation spectra have a generic quasi-universal form that justifies these operations. The procedures outlined here are vastly simpler than performing a non-linear regression scheme and most importantly, yield parameters that closely fit the experimental data. Simplicity with respect to experimentation, computation and interpretation is the principal objective of developing the Cole-Cole characterization method.

We outline our method to determine the Cole-Cole model parameters below. The fundamental experimental data used in the Cole-Cole model are the dynamic viscosity (or moduli) and it is from these data that we shall derive the parameter values. The key step in the parameter determination is the evaluation of the characteristic relaxation time, $\lambda_{0}$. The real and imaginary components of the complex viscosity according to the Cole-Cole model are, 
$\eta^{\prime}(\omega)=\frac{\eta_{0}\left[1+(\omega \lambda)^{(1-\alpha)} \sin \left(\frac{\alpha \pi}{2}\right)\right]}{1+2(\omega \lambda)^{(1-\alpha)} \sin \left(\frac{\alpha \pi}{2}\right)+(\omega \lambda)^{2(1-\alpha)}}$

and

$\eta^{\prime \prime}(\omega)=\frac{\eta_{0}(\omega \lambda)^{(1-\alpha)} \cos \left(\frac{\alpha \pi}{2}\right)}{1+2(\omega \lambda)^{(1-\alpha)} \sin \left(\frac{\alpha \pi}{2}\right)+(\omega \lambda)^{2(1-\alpha)}}$

respectively.

The maximum in the imaginary dynamic viscosity curve occurs when, $\left.\frac{\mathrm{d} \eta^{\prime}}{\mathrm{d} \omega}\right|_{\omega=\omega_{\mathrm{p}}}=0$. The reciprocal of the frequency at which this maximum occurs defines the characteristic relaxation time (Marine et al. 1975; Montfort et al. 1984; Montfort 1976).

$\lambda_{0}=\frac{1}{\omega_{\mathrm{p}}}$

The real and imaginary components at the characteristic frequency provide the following expressions for the zero shear viscosity parameter;

$\eta_{0}=2 \eta^{\prime}\left(\omega_{\mathrm{p}}\right)=2 \eta^{\prime \prime}\left(\omega_{\mathrm{p}}\right) \tan \delta\left(\omega_{\mathrm{p}}\right)$

Finally, the $\alpha$ parameter is defined in terms of the phase angle evaluated at the characteristic frequency,

$\tan \delta\left(\omega_{\mathrm{p}}\right)=\cot \left[(1-\alpha) \frac{\pi}{4}\right]=\frac{\eta^{\prime}\left(\omega_{\mathrm{p}}\right)}{\eta^{\prime \prime}\left(\omega_{\mathrm{p}}\right)}$
Thus, once the characteristic frequency is evaluated, the other parameters follow immediately.

The so-called number average and weight average relaxation times are defined below:

$\lambda_{\mathrm{n}}=\frac{\int_{-\infty}^{\infty} \lambda H(\lambda) \mathrm{d} \ln \lambda}{\int_{-\infty}^{\infty} H(\lambda) \mathrm{d} \ln \lambda}=\frac{\eta_{0}}{G_{\mathrm{n}}^{0}}$

$\lambda_{\mathrm{w}}=\frac{\int_{-\infty}^{\infty} \lambda^{2} H(\lambda) \mathrm{d} \ln \lambda}{\int_{-\infty}^{\infty} \lambda H(\lambda) \mathrm{d} \ln \lambda}=\eta_{0} J_{\mathrm{e}}^{0}$

The dispersion in the relaxation spectrum is often characterized by the ratio of the weight average relaxation time to the number average relaxation time, $\lambda_{\mathrm{w}} / \lambda_{\mathrm{n}}$. Since dispersion in the relaxation spectrum and dispersion in the molecular weight distribution go hand in hand, $\lambda_{\mathrm{w}} / \lambda_{\mathrm{n}}$ correlates with $M_{\mathrm{w}} / M_{\mathrm{n}}$. Typically, the value of the Cole-Cole characteristic relaxation $\lambda_{0}$ lies between these characteristic relaxation times (Montfort et al. 1984).

$\lambda_{\mathrm{w}}>\lambda_{0}>\lambda_{\mathrm{n}}$

For polymers where the molecular weight distribution has a single peak, there is a unique maximum in the complex viscosity versus frequency. For more complex systems with multiple maxima in the molecular weight distribution, multiple characteristic frequencies may exist. 